\title{
A novel RNA-guided RNA-targeting CRISPR tool
}

\author{
Dandan Zhang, Zhenxiang Li, Bingyu Yan \& Jian-Feng Li* \\ Key Laboratory of Gene Engineering of the Ministry of Education, State Key Laboratory of Biocontrol, Guangdong Provincial Key Laborato- \\ ry of Plant Resources, School of Life Sciences, Sun Yat-sen University, Guangzhou 510275, China
}

Received June 20, 2016; accepted June 28, 2016; published online July 18, 2016

Citation: Zhang, D., Li, Z., Yan, B., and Li J.F. (2016). A novel RNA-guided RNA-targeting CRISPR tool.. Sci China Life Sci 59, 854-856. doi: 10.1007/s11427-016-0192-5

Clustered regularly interspaced short palindromic repeats (CRISPR)/CRISPR-associated protein (Cas) technology has emerged as a programmable RNA-guided DNA-targeting tool for sequence-specific genome editing in diverse organisms. The most frequently used CRISPR/Cas system, which is based on the bacterium Streptococcus pyogenes Cas9 (SpCas9) endonuclease, has been repurposed into a suite of versatile tools for numerous DNA-manipulating activities beyond genome editing (Jiang and Marraffini, 2015). However, progress in converting the CRISPR/SpCas9 system into a sequence-specific RNA-manipulating platform has been limited. A recent study published in Science by Abudayyeh and colleagues (Abudayyeh et al., 2016) has identified Leptotrichia shahii C2c2 (LshC2c2) to be a novel programmable RNA-targeting endoribonuclease with great potential to reshape the landscape of future RNA research.

Current CRISPR/SpCas9 technology was originally developed from the bacterial type-II CRISPR/Cas adaptive immune system, which evolves to purge invading bacteriophage and plasmid DNA. In this technology, a single synthetic guide RNA (sgRNA) containing a 20-nt guide (also known as spacer) sequence is used to target the SpCas9 nuclease to an intended genomic $\mathrm{N}_{20} \mathrm{NGG}$ ( $\mathrm{N}$ for any base) sequence through RNA-DNA base pairing (Figure 1A). "NGG" is known as the protospacer adjacent motif (PAM), which is absolutely required for SpCas9 target recognition. Subsequent DNA cleavage by SpCas9 generates a DNA double-strand break, which in turn leads to target gene mutagenesis or replacement as a result of DNA repair. As the

*Corresponding author (email: lijfeng3@mail.sysu.edu.cn)
DNA-targeting specificity is determined by RNA-DNA complementarity, the CRISPR/SpCas9 system exhibits unprecedented simplicity and multiplexability compared to earlier genome editing tools (e.g., zinc-finger nucleases (ZFNs) and transcription-activator like effector nucleases (TALENs)) that are based on protein-DNA interaction. These striking merits have also stimulated the conversion of nuclease-dead SpCas9 (dCas9) into a programmable DNA-binding platform. When fused with different effector domains (e.g., a fluorescent protein or transcriptional or epigenetic regulatory domains), the dCas9-effector chimera empowers specific labeling or regulatory activities at specific DNA sequences in a genome (Dominguez et al., 2016).

RNA plays indispensable roles within cells, as building blocks (e.g., mRNA, tRNA, and rRNA) or regulatory components (e.g., miRNA and lncRNA) of gene transcription and protein translation machineries, as well as genetic information carriers (i.e., viral RNA). Therefore, it is highly desirable to control the activities of RNA molecules in a sequence-specific manner. A common strategy for targeted RNA manipulation involves engineering of RNA-binding proteins (e.g., PUF proteins) that have well-defined RNArecognition modules but unsatisfactory RNA-targeting specificities (Nelles et al., 2015). The ease of the CRISPR/ SpCas9 system in manipulating DNA has also motivated its modification for RNA manipulation (O'Connell et al., 2014). Cleavage of single-strand RNA (ssRNA) by the CRISPR/SpCas9 system was achieved in vitro when a PAM-containing oligonucleotide, termed a PAMmer, was supplied to complement the target ssRNA (Figure 1B). Furthermore, a chimera dCas9-fluorescent protein was suc- 

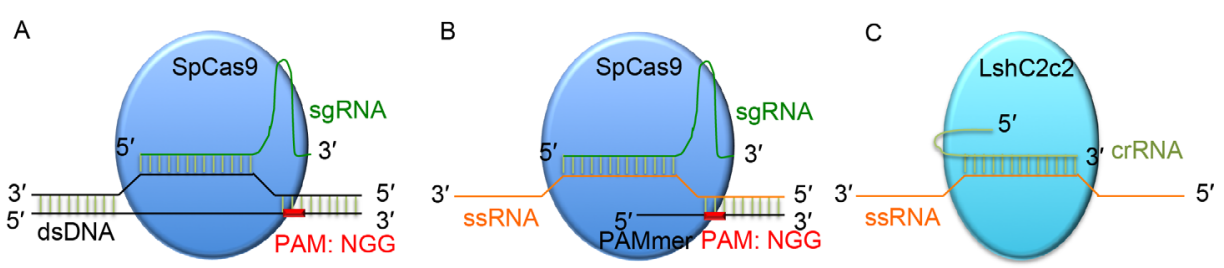

Figure 1 CRISPR/Cas systems for DNA or RNA targeting. A, Diagram of the SpCas9-sgRNA complex targeting double-strand DNA (dsDNA). PAM, protospacer adjacent motif. B, Diagram of the SpCas9-sgRNA complex targeting ssRNA in the presence of a PAMmer. Note that this system is also known as the RCas9 system. C, Diagram of the LshC2c2-crRNA complex targeting ssRNA.

cessfully implemented to image the trafficking of specific mRNAs in human cells with the assistance of PAMmers (Nelles et al., 2016). However, for this so-called RNAtargeted Cas9 (RCas9) system, efficient delivery of PAMmers into whole animals or plants to facilitate RNA targeting remains a gigantic roadblock. Interestingly, the bacterium Francisella novicida Cas9 (FnCas9), an ortholog of SpCas9, was also found to associate with mRNA in bacteria (Sampson et al., 2013) and with viral RNA in human cells (Price et al., 2015). However, this system is unable to distinguish RNA from DNA targets.

The work by Abudayyeh and colleagues now shows that the CRISPR/LshC2c2 system, which is developed from the type-VI CRISPR/Cas adaptive immune system in L. shahii, can be engineered into a programmable RNA-guided RNA-targeting system (Figure 1C), thus opening up a new avenue for RNA targeting. The authors were attracted to LshC2c2 because, as the only effector in this CRISPR/Cas system, it shows no homology to DNA nucleases, but instead possesses two Higher Eukaryotes and Prokaryotes Nucleotide-binding (HEPN) domains, which exist in many RNases. Indeed, co-expression of LshC2c2 and a CRISPR RNA (crRNA) containing a 28-nt spacer targeting MS2 phage RNA could confer viral resistance to $E$. coli. Detailed characterization of purified LshC2c2 in vitro showed that LshC2c2 only cleaves ssRNA, but not any other form of nucleic acid, upon recognizing an $\mathrm{N}_{28} \mathrm{H}$ ( $\mathrm{H}$ for $\mathrm{A}, \mathrm{C}$, and $\mathrm{U}$ ) target sequence complementary to the crRNA spacer. The authors further demonstrated that LshC2c2 cleaves exposed regions (e.g., loop regions) within ssRNA outside the target sequence, with a high preference for $\mathrm{U}$ residues. The HEPN domains of LshC2c2 appear to be responsible for RNA cleavage, because mutation of a putative catalytic residue within either HEPN domain eliminates RNA cleavage activity. The authors next demonstrated that the spacer length of an effective crRNA should be at least $22 \mathrm{nt}$, and that the stem-loop structure of the crRNA is critical for target RNA recognition. Using a 28-nt spacer, the authors investigated the RNA-targeting specificity of LshC2c2 both in vitro and in E. coli, concluding that single mismatches across the spacer can be tolerated but two mismatches distributed in the central region of the spacer can dramatically compromise target RNA cleavage. Finally, the authors successfully applied the CRISPR/LshC2c2 system to cleave target
mRNA in E. coli. Notably, they observed remarkable growth arrest of $E$. coli, correlated with efficient knockdown of the target mRNA. They provided evidence that the LshC2c2-crRNA complex, once activated by target ssRNA, could cleave other ssRNA non-specifically, which may lead to cytotoxicity and subsequent growth inhibition.

The aforementioned study was carried out exclusively in E. coli and in vitro. It would be compelling to test whether this system could effectively cleave ssRNA in eukaryotic cells. It might be possible that the cytotoxicity of target-activated LshC2c2 is limited to E. coli, as the cytotoxicity of SpCas9 has only been witnessed in single-cell organisms, such as E. coli, Agrobacterium, and Chlamydomonas, but never in mammalian or plant cells. If this were not true, application of the CRISPR/LshC2c2 system for degrading target mRNA or other non-coding RNA would be problematic. We hope that comparison of the crystal structures of target-activated and idling LshC2c2-crRNA complexes will offer guidance for creating an improved variant of LshC2c2 with tightly controllable cleavage activity. Transformation of the CRISPR/LshC2c2 system into a weapon against RNA viruses causing human and plant diseases (Zhang et al., 2015) represents another attractive possibility. When comparing the CRISPR/LshC2c2 system to current gene silencing or antiviral technologies, such as RNAi and artificial miRNA (amiRNA), it is still too early to determine whether the former will render the latter obsolete. For instance, the minimal spacer length $(\geqslant 22 \mathrm{nt})$ required by LshC2c2 limits its utility for cleaving small non-coding RNA (e.g., 21-nt miRNA), which can be destroyed using complimentary amiRNA.

Compared to wild type, ribonuclease-dead dC2c2 may be more valuable as a programmable sequence-specific RNAbinding platform, assuming that the target RNA-dC2c2 association is frozen without cleavage to promote the release of target RNA substrate and re-binding of another nontarget RNA. It is conceivable that $\mathrm{dC} 2 \mathrm{c} 2$ could be useful in the following applications: (i) visualization of localization and trafficking of a specific mRNA in a cell or labeling specific cells expressing a certain mRNA via fluorescently tagged $\mathrm{dC} 2 \mathrm{c} 2$, (ii) sequestration of a specific mRNA at a desired subcellular locus via co-localization with $\mathrm{dC} 2 \mathrm{c} 2$, (iii) boosting protein production from a specific mRNA, using a $\mathrm{dC} 2 \mathrm{c} 2$ fusion with an RNA-stabilizing protein or 
translation-enhancing factor, (iv) alteration of the splicing pattern of a specific mRNA by fusion of $\mathrm{dC} 2 \mathrm{c} 2$ with a splicing factor, (v) C-to-U editing of a specific mRNA using a dC2c2-cytidine deaminase chimera, (vi) pulling down a specific RNA to identify its associated proteins, and (vii) evaluating the interaction between a candidate protein and a specific RNA by BiFC with $\mathrm{dC} 2 \mathrm{c} 2$ and the candidate protein fused respectively to split fluorescent proteins. It is noteworthy that effector domains can also be recruited as a fusion to the MS2 coat protein (MCP) through a MCP-binding aptamer embedded within the stem-loop region of crRNA, as long as aptamer insertion does not impair the stem-loop structure. As Abudayyeh et al. noted, the secondary structure of the target RNA can greatly affect the targeting efficiency of the LshC2c2-crRNA complex (Abudayyeh et al., 2016), presumably owing to the variable accessibility of different target sequences within a complicatedly folded ssRNA. Therefore, it is most desirable to screen multiple target sequences from the same target RNA to identify the most effective one for a given application.

The elegance of $\mathrm{LshC} 2 \mathrm{c} 2 / \mathrm{dC} 2 \mathrm{c} 2$ performance in vivo will ultimately rely on its targeting specificity, which awaits systematic validation and has room for improvement. One strategy for specificity improvement is to reduce the length of the crRNA spacer from $28 \mathrm{nt}$ to an optimal length that allows sufficient affinity between crRNA and the target RNA while substantially increasing the sensitivity of the system to mismatches within the crRNA-ssRNA duplex. A truncated sgRNA strategy has been adopted to reduce off-target effects in the CRISPR/SpCas9 system (Fu et al., 2014). One caveat is that the crRNA-ssRNA duplex may induce the synthesis of double-strand RNA within eukaryotic cells, which could in turn trigger secondary RNAi events, leading to unwanted off-target silencing. Shortening the spacer length of the crRNA could help minimize this possibility. Another strategy to improve the targeting specificity of LshC2c2/dC2c2 is based on structural insights. For example, structure-guided engineering has been applied to generate an enhanced SpCas9 that exhibits extremely high DNA-targeting specificity (Slaymaker et al., 2016). Identification of a new bacterial antiviral RNase analogous to LshC2c2 with better targeting specificity and little or no promiscuous cleavage activity is also a possibility. Consid- ering the incredible pace of development of CRISPR technology, from the CRISPR/SpCas9 system to the RCas9 system to the CRISPR/LshC2c2 system (Figure 1), the next significant advance in programmable RNA targeting should be on the horizon, which will benefit all RNA-related research in biology and medicine.

Compliance and ethics The author(s) declare that they have no conflict of interest.

Acknowledgements This work was supported by the National Natural Science Foundation of China (31522006), and China's Thousand Young Talents Program to Jian-Feng Li.

Abudayyeh, O.O., Gootenberg, J.S., Konermann, S., Joung, J., Slaymaker, I.M., Cox, D.B.T., Shmakov, S., Makarova, K.S., Semenova, E., Minakhin, L., Severinov, K., Regev, A., Lander, E.S., Koonin, E.V., and Zhang, F. (2016). C2c2 is a single-component programmable RNA-guided RNA-targeting CRISPR effector. Science, doi: 10.1126/science.aaf5573.

Dominguez, A.A., Lim, W.A., and Qi, L.S. (2016). Beyond editing: repurposing CRISPR-Cas9 for precision genome regulation and interrogation. Nat Rev Mol Cell Biol 17, 5-15.

Fu, Y., Sander, J.D., Reyon, D., Cascio, V.M., and Joung, J.K. (2014). Improving CRISPR-Cas nuclease specificity using truncated guide RNAs. Nat Biotechnol 32, 279-284.

Jiang, W., and Marraffini, L.A. (2015). CRISPR-Cas: new tools for genetic manipulations from bacterial immunity systems. Annu Rev Microbiol 69, 209-228.

Nelles, D.A., Fang, M.Y., Aigner, S., and Yeo, G.W. (2015). Applications of Cas9 as an RNA-programmed RNA-binding protein. Bioessays 37, 732-739.

Nelles, D.A., Fang, M.Y., O’Connell, M.R., Xu, J.L., Markmiller, S.J., Doudna, J.A., and Yeo, G.W. (2016). Programmable RNA tracking in live cells with CRISPR/Cas9. Cell 165, 488-496.

O’Connell, M.R., Oakes, B.L., Sternberg, S.H., East-Seletsky A., Kaplan, M., and Doudna, J.A. (2014). Programmable RNA recognition and cleavage by CRISPR/Cas9. Nature 516, 263-266.

Price, A.A., Sampson, T.R., Ratner, H.K., Grakoui, A., and Weiss, D.S. (2015). Cas9-mediated targeting of viral RNA in eukaryotic cells. Proc Natl Acad Sci USA 112, 6164-6169.

Sampson, T.R., Saroj, S.D., Llewellyn, A.C., Tzeng, Y.L., and Weiss, D.S. (2013). A CRISPR/Cas system mediates bacterial innate immune evasion and virulence. Nature 497, 254-257.

Slaymaker, I.M., Gao, L., Zetsche, B., Scott, D.A., Yan, W.X., and Zhang, F. (2016). Rationally engineered Cas9 nucleases with improved specificity. Science $351,84-88$.

Zhang, D., Li, Z., and Li, J.F. (2015). Genome editing: new antiviral weapon for plants. Nat Plants 1, 15146.

Open Access This article is distributed under the terms of the Creative Commons Attribution License which permits any use, distribution, and reproduction in any medium, provided the original author(s) and source are credited. 